\title{
Bilateral Laparoscopic Partial Adrenalectomy: A Case Presentation and Review of the Literature
}

\author{
Sean Satey ${ }^{\mathrm{a}}$, Nathan Richards ${ }^{\mathrm{a}}$, Fred Brody ${ }^{\mathrm{a}, \mathrm{b}}$
}

\begin{abstract}
Laparoscopic adrenalectomy is the standard of care for benign adrenal diseases. Partial adrenalectomy has been performed for various tumors to preserve adrenal function while avoiding steroid supplements postoperatively. This article discusses a patient that underwent bilateral laparoscopic partial adrenalectomies for bilateral pheochromocytomas and reviews the literature on partial adrenalectomy. A 40-year-old woman with a recent diagnosis of hypertension and an emergent Caesarian section for pre-eclampsia presented to an outside clinic with labile blood pressure, diaphoresis, palpitations, and headache. Physical examination was unremarkable with no bruits appreciated in the neck or abdomen. Medical management of her hypertension was initiated with minimal response. Ultimately, a 24-h urine demonstrated elevated urinary metanephrine and normetanephrine levels consistent with a pheochromocytoma. An abdomen and pelvis computed tomogram revealed bilateral, discrete, solid adrenal masses measuring $3.9 \times$ $3.3 \mathrm{~cm}$ and $2.3 \times 2.7 \mathrm{~cm}$ on the left and right, respectively. Selective adrenal venous sampling confirmed bilateral biochemically active pheochromocytomas. Bilateral laparoscopic partial adrenalectomies were performed using laparoscopic staplers to divide the tumor from the normal gland. Both adrenal veins were preserved. Glucocorticoid function was monitored by serial cortisol measurements and glucocorticoid supplementation was initiated on postoperative day one with a 4-week taper. At 1-year follow-up, she remains asymptomatic with a normal blood pressure, cortisol levels, and urine catecholamines. Laparoscopic partial adrenalectomies for bilateral pheochromocytomas are safe and effective. Partial resections preserve adrenal function while eradicating long-term steroid requirements.
\end{abstract}

Keywords: Bilateral laparoscopic partial adrenalectomy; Pheochromocytoma; Steroid; Operative technique

\footnotetext{
Manuscript accepted for publication February 18, 2014

aDivision of General Surgery, Department of Surgery, George Washington University School of Medicine and Health Sciences, 2150 Pennsylvania Avenue, NW, Suite 6B, Washington, DC 20037, USA

${ }^{\mathrm{b}}$ Corresponding author: Fred Brody, 2150 Pennsylvania Avenue, NW, Suite 6B, Washington, DC 20037, USA. Email: fbrody@mfa.gwu.edu
}

doi: http://dx.doi.org/10.14740/jmc1708w

\section{Introduction}

Laparoscopy is the current standard surgical approach for non-malignant tumors of the adrenal glands [1]. In 1999, Guazzoni et al demonstrated the myriad advantages of laparoscopy for adrenal disorders including decreased length of stay, decreased post-operative analgesia and lower rates of minor and major complications [2-4]. Open adrenalectomies are now reserved for large tumors or locally invasive adrenocortical cancer requiring lymph node or adjacent organ resection [5]. However, even tumors greater than $10 \mathrm{~cm}$ in size have been resected laparoscopically depending on surgeon experience.

Recently, laparoscopic partial adrenalectomy has been used for functional and non-functional benign adrenal tumors. This approach may preserve adrenocortical function and catecholamine excretion while avoiding post-operative chronic steroid replacement [6]. Walz et al described a series of laparoscopic partial adrenalectomies in 2004. They documented a mean tumor size of $2.8 \mathrm{~cm}$, mean operative time of $80 \mathrm{~min}$, and mean blood loss of $29 \mathrm{~mL}$. All patients had a biochemical cure without evidence of recurrence at 51-month follow-up [7]. This paper discusses a patient who underwent bilateral laparoscopicpartial adrenalectomies for bilateral pheochromocytomas and reviews the literature on partial adrenalectomy.

\section{Case Report}

The patient is a previously healthy 40 -year-old woman who was diagnosed with hypertension in November 2010. She underwent an emergency C-section in June 2011 for preeclampsia associated with sweating, palpitations, and headaches. After delivery, her symptoms persisted and blood pressure remained labile with a peak of $220 / 150 \mathrm{mmHg}$. Her physical examination was unremarkable with no bruits in the neck or abdomen. Anti-hypertensive medication was started with amlodipine/olmesartanmedoxomil $10 / 40 \mathrm{mg}$ once daily. In October 2011, she developed eye redness and pain, and was diagnosed with posterior uveitis. Initial treatment 


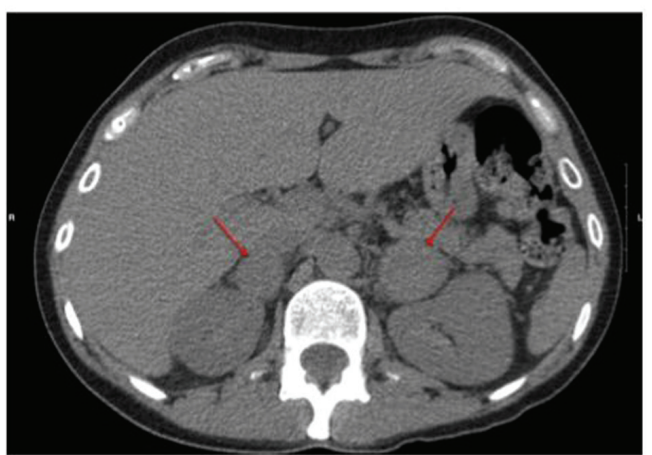

Figure 1. Pre-operative CT scan showing bilateral adrenal masses as highlighted by arrows.

with ophthalmic steroid drops was suboptimal. The patient was then switched to a 3-week oral steroid regimen, with full resolution of her uveitis. The combination of hypertension and uveitis raised the possibility of Takayasu arteritis. Rheumatologic evaluation demonstrated normal ANA, ANCA, HLA-B27, ACE, eGFR, and liver function tests. Her urine studies were void of hyaline casts, white blood cells, red blood cells, and protein. She was noted to have a persistent blood pressure of 190/110 $\mathrm{mmHg}$ in both arms despite the addition of hydrochlorothiazide $12.5 \mathrm{mg}$ daily. Due to the persistent hypertension, an angiogram was performed to assess her great vessels and renal arteries. The angiogram was normal without evidence of stenosis.

A 24-h urine revealed markedly elevated levels of metanephrines $(513 \mu \mathrm{g} / 24 \mathrm{~h}, \mathrm{nml}: 45$ - 290) and normetanephrines $(9,923 \mu \mathrm{g} / 24 \mathrm{~h}, \mathrm{nml}: 82$ - 500). Serum aldosterone (34.5 ng/ $\mathrm{dL}, \mathrm{nml}: 0.0$ - 30.0) and plasma renin $(107.02 \mathrm{ng} / \mathrm{mL} / \mathrm{h}, \mathrm{nml}$ : 0.29 - 3.70) were also elevated. An abdomen and pelvis computed tomography (CT) revealed bilateral, discrete, solid adrenal masses. The left and right adrenal masses measured 3.9 $\times 3.3 \mathrm{~cm}$ and $2.3 \times 2.7 \mathrm{~cm}$, respectively. The remainder of the CT was normal without evidence of hepatic or pancreatic lesions (Fig. 1). Selective adrenal venous sampling confirmed bilateral functional pheochromocytomas. Calcitonin and parathyroid hormone levels were normal. Based on this work up, bilateral laparoscopic partial adrenalectomies were planned. Four weeks pre-operatively, the patient received alpha-adrenergic blockade with phenoxybenzamine. Once adequate alpha-adrenergic blockade had been attained, betaadrenergic blockade with metoprolol was initiated 3 days preoperatively.

After the induction of adequate general endotracheal anesthesia, the patient was placed in the right lateral decubitus position. A Veress needle was inserted along the left costal margin and the abdomen was insufflated to $15 \mathrm{mmHg}$ with carbon dioxide. A $10 \mathrm{~mm}$ Visiport (Covidien, Mansfield, MA) was then inserted. Diagnostic laparoscopy did not discern any abnormal pathology. Three $5 \mathrm{~mm}$ ports were then placed along the costal margin. The splenic flexure of the

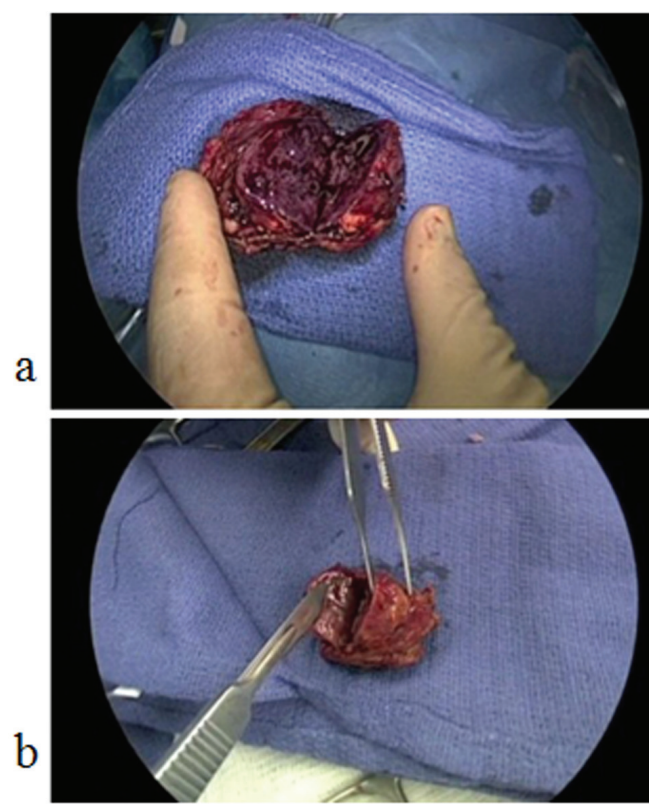

Figure 2. (a) Bi-valved left pheochromoctyoma with negative margins and circumferentially intact; (b) Bi-valved right pheochromoctyoma with negative margins and circumferentially intact.

colon was mobilized inferiorly along with the distal transverse colon. The spleen and pancreas were rotated medially by incising the peritoneal lining approximately $1 \mathrm{~cm}$ from the lateral aspect of the spleen. The lateral border of the left adrenal gland was mobilized from the fat pad overlying Gerota's fascia. The peritoneal lining along the medial aspect of the left adrenal gland was incised and the adrenal gland was elevated from the retroperitoneum. By elevating the gland, the left adrenal vein was identified in a cephalad and caudal direction. The contour of the tumor was appreciated along with the normal adrenal gland. A laparoscopic stapler was applied along the contour of the tumor and a 5 $\mathrm{mm}$ margin was obtained from the normal adrenal gland. Two staplers were utilized and the tumor was inserted in a specimen bag. Hemostasis was maintained along the staple line with approximately $10 \mathrm{~mL}$ of Tisseel (Baxter, Deerfield, IL). The tumor was then extracted from the abdomen after increasing the size of the $10 \mathrm{~mm}$ port. The specimen was circumferentially intact with grossly negative macroscopic margins (Fig. 2a). All ports were then closed.

The patient was then rotated into the left lateral decubitus position. Abdominal access was obtained in a similar fashion with the Veress needle and three $5 \mathrm{~mm}$ ports along the costal margin. The liver was retracted superiorly and the inferior border of the adrenal gland was dissected from Gerota's fascia. The medial aspect of the adrenal gland was dissected from the inferior vena cava and the right adrenal vein was kept intact during the dissection. The adrenal tumor was identified inferiorly and laterally and the contour of the 
normal gland was identified. The normal gland and tumor were elevated from the retroperitoneum in a similar fashion. Two laparoscopic staplers were utilized to divide the normal adrenal gland and the tumor with a $5 \mathrm{~mm}$ margin. The specimen was placed in a bag and extracted from the wound. The staple line along the adrenal gland was hemostatically intact and $10 \mathrm{~mL}$ of Tisseel were applied again along the staple line. The specimen was circumferentially intact with macroscopically negative margins (Fig. 2b). All ports were closed and the patient was extubated.

The patient was started on a liquid diet on post-operative day (POD) one and advanced to a regular diet on POD two. Her morning cortisol level was $2.9 \mu \mathrm{g} / \mathrm{dL}$ (nml: 4.5 - 23) on POD one. Glucocorticoid supplementation was initiated with $10 \mathrm{mg}$ prednisone in the morning and $10 \mathrm{mg}$ in the evening for 7 days. She was discharged on the same dose of prednisone. Morning cortisol levels were measured on POD 7,21 , and 28. On POD seven, her morning cortisol level was $3.9 \mu \mathrm{g} / \mathrm{dL}$ and her prednisone dose was tapered to $5 \mathrm{mg}$ twice daily for 14 days. On POD 21, her morning cortisol was 15 $\mu \mathrm{g} / \mathrm{dL}$ and her prednisone dose was again tapered to $5 \mathrm{mg}$ once daily for seven days. On POD 28, her morning cortisol was $12 \mu \mathrm{g} / \mathrm{dL}$ and her steroids were discontinued. Thereafter, all cortisol levels were normal.

Post-operatively, she remained on her pre-operative regimen of phenoxybenzamine $10 \mathrm{mg}$ twice daily with a maximum blood pressure of $140 / 100 \mathrm{mmHg}$ on POD two. On POD 21, the dosage was tapered to $10 \mathrm{mg}$ daily with a blood pressure of 130/90 $\mathrm{mmHg}$. On POD 28, the phenoxybenzamine was discontinued with a blood pressure of $120 / 80$ mmHg. In addition, VHL receptor testing and RET protooncogene assays were performed with no evidence of mutations. Final surgical pathology was consistent with bilateral benign pheochromocytomas with intact capsules.

Her 1-year evaluation was notable for a morning cortisol of $13 \mu \mathrm{g} / \mathrm{dL}$ and blood pressure of $124 / 88 \mathrm{mmHg}$ without steroids or anti-hypertensive medications. Laboratory testing of urinary biomarkers were all normal.

\section{Discussion}

Laparoscopic bilateral partial adrenalectomies may be safely and effectively performed for bilateral pheochromocytomas. Pre-operative management of pheochromocytoma involves two phases. The first phase involves adrenergic blockade while the second involves volume expansion. Currently, there is no universally accepted method of surgical preparation for pheochromocytomas. Accepted preoperative preparations include combined alpha- and beta-adrenergic blockade, calcium channel blockers (CCB), or metyrosine. Nicardipine, a common CCB, is primarily used in patients that suffer from intolerable side effects of alpha- or beta-adrenergic blockers [8]. Metyrosine, an inhibitor of catechol- amine synthesis, may be used in patients that cannot take alpha- or beta-blockers due to cardiopulmonary issues or drug intolerance. In this patient, alpha-adrenergic blockade via phenoxybenzamine was started four weeks pre-operatively. Once her pre-operative intravascular space was expanded with alpha blockade, beta-adrenergic blockade via metoprolol was started three days pre-operatively.

The surgical approach for open and laparoscopic adrenalectomy varies. In open procedures, the adrenal gland may be excised either through an extra-peritoneal approach (dorsal or flank incision) or an anterior transperitoneal approach (subcostal). Laparoscopic approaches include a transperitoneal or a retroperitoneal (lateral or posterior) technique. Most general surgeons use a lateral transperitoneal approach as they are more familiar with the visceral orientation. The lateral transabdominal approach utilizes gravity to retract the spleen and pancreas medially and provides a larger working space versus the retroperitoneal space. Conversely, the retroperitoneal approach may prevent injury to intra-abdominal organs and the smaller space may tamponade post-operative oozing. As well, the retroperitoneal technique provides the most direct access to the adrenal gland which may be useful in morbidly obese patients [9].

In this patient and throughout the literature, partial adrenalectomy can preserve biochemical function of the adrenal gland [10]. Theoretically, the adrenal vein should remain intact with a portion of the adrenal gland. The smallest amount of intact adrenal gland required for normal physiology is unknown. Brauckhoff et al reviewed 10 patients that underwent subtotal adrenalectomies with preservation of less than a third of the adrenal gland and transection of the main adrenal vein. After the surgical procedure, these investigators followed patients with adrenocorticotropine hormone (ACTH) testing to confirm normal adrenocortical function. Brauckhoff et al concluded that venous drainage via the main adrenal vein was not critical for adrenocortical function and an estimated one-third remnant of adrenal tissue was sufficient for normal adrenocortical function [11].

From an anatomical standpoint, Parnaby et al studied 6 cadaveric and 164 laparoscopic adrenal cases to illustrate the adrenal blood supply with relevance to laparoscopic partial adrenalectomies. The study documented numerous small veins along the periphery of the gland called venae comitantes. Based on their findings, Parnaby et al recommend that excessive adrenal gland mobilization should not be performed in order to avoid disruption of the venous tributaries [12]. Therefore, the main adrenal vein may be ligated during a partial adrenalectomy as venous drainage may be maintained through the tributaries. Other studies suggest that preservation of the adrenal vein is important for normal adrenal physiology. Roukounakis et al reviewed seven patients that underwent laparoscopic partial adrenalectomies. They preserved venous drainage via the main adrenal vein during each partial resection to ensure a functioning adre- 
nal remnant. In theory, an intact main adrenal vein avoids venous congestion [13]. Unless immediately adjacent to the tumor, the main venous drainage should be kept intact. In addition, the connective tissue around the remnant adrenal tissue should remain intact to preserve arterial supply and prevent possible torsion [14]. In this patient, the main vein was kept intact bilaterally. The segmental anatomy of the adrenal gland lends itself to partial division of its arterial supply. Depending on the portion of adrenal tissue involved, the respective arterial supply may be ligated without interfering with the overall arterial supply and functionality of the remaining adrenal tissue [15].

Adrenal division may be performed using several modalities including electrocautery, ultrasonic energy, clips, shears or staplers. Since the adrenal gland is highly vascular, laparoscopic partial adrenalectomy poses a significant risk of bleeding. A laparoscopic stapler may minimize bleeding due to three uniform staple lines. This technique was highly effective in this patient. As well, laparoscopic staplers may decrease operative times [16-18]. On the other hand, Jeschke et al used a combination of bipolar energy and endoshears to obtain a margin of normal tissue with the aid of intraoperative ultrasound [19]. Also, several studies illustrate the utility of ultrasonic therapy to control bleeding during adrenal parenchymal division $[18,20,21]$. Ultimately, the technique of parenchynmal division depends on surgeon preference and adrenal pathology. Post-operatively, patients are hospitalized for one to two days after an uncomplicated laparoscopic partial adrenalectomy versus three to five days for an open adrenalectomy [22].

No guidelines are currently available for post-operative management following a partial adrenalectomy [23]. Glucocorticoid and mineralocorticoid replacement therapy may be given to patients with subclinical cortisol and sodium levels postoperatively. Measuring morning cortisol levels is the most common modality for determining replacement therapy. The steroids administered most commonly include prednisone, hydrocortisone, methylprednisolone, and fludrocortisone. In this patient, her glucocorticoid (prednisone) requirements were tapered successfully during a four-week postoperative course. Laboratory values (morning cortisol and urinary metabolites) at one-year follow up remained at physiologic levels. In Brauckhoff's previously mentioned article, all ten patients had normal serum cortisol levels in the early postoperative period. However, six of the ten patients had pathologically elevated plasma ACTH levels (mean, $55 \pm 42 \mathrm{pmol} / \mathrm{mL}$; normal, < 10.1) but no patient required steroid supplementation. At one-year follow up, all patients had normal ACTH test results. They concluded that during the early post-operative period, careful observation of patients without exogenous steroid supplementation may be performed [11].

Although adrenal tumors are predominantly sporadic, adrenal neoplasms may be associated with genetic syn- dromes. These include multiple endocrine neoplasia type 2 (MEN2), Von Hippel-Lindau (VHL) disease, neurofibromatosis type 1 (NF1), and mutations in succinate dehydrogenase (SDH) subunits $\mathrm{B}, \mathrm{D}$, and $\mathrm{C}$. The literature suggests that all patients with evidence of pheochromocytomas should undergo genetic testing to detect a potential underlying syndrome. This is particularly true for patients with bilateral adrenal tumors.

MEN2 involves a genetic defect in the RET proto-oncogene on chromosome 10. All organs that express the RET gene may result in tumor formation. These organs include the thyroid gland (medullary thyroid cancer), adrenal glands (pheochromocytoma), and parathyroid glands (primary hyperparathyroidism). Within the MEN2 syndrome, clinical symptoms associated with pheochromocytomas usuallymanifest before evidence of thyroid or parathyroid disease. Pheochromocytomas associated with MEN2 are almost always bilateral while sporadic etiologies tend to be unilateral. Genetic testing for the patient and family members is imperative with the following scenarios: 1) MEN2 is clinically proven, 2) medullary thyroid cancer or pheochromocytoma is found in patients with a family member with medullary thyroid carcinoma or pheochromocytoma, 3) sporadic MEN2 tumor and young age ( $<35$ years old $), 4)$ multicentric tumor in one organ, or 5) bilateral organs (as in this patient) or two different organs are affected.

Von Hippel-Lindau (VHL) disease is an inherited, autosomal dominant syndrome that predisposes individuals to benign and malignant neoplasms throughout the body. VHL disease has been noted in 1:36,000 people. Common tumors associated with VHL disease include pheochromocytomas, central nervous system hemangioblastomas, clear cell renal carcinomas, pancreatic neuroendocrine tumors, middle ear endolymphatic sac tumors, and pancreatic cysts [24]. VHL disease is traditionally divided into Type I and II based on the likelihood of pheochromocytoma development. Type II is the most common. Genetic testing may be performed via peripheral blood lymphocytes, DNA sequencing, or VHL gene analysis with $100 \%$ sensitivity and specificity.

Based on the above review, laparoscopic partial adrenalectomies for bilateral pheochromocytomas are safe and effective. Margin negative resection is imperative. Partial resections for bilateral pheochromocytomas preserve adrenal function and can significantly impact patient quality of life by eradicating the need for lifelong glucocorticoid requirements while alleviating the associated symptoms of a pheochromocytoma. Finally, genetic testing for bilateral pheochromocytomas should be performed for patients and their respective family members.

\section{References}

1. Janetschek G, Marberger M. Laparoscopic surgery in 
urology. Curr Opin Urol. 2000;10(4):351-357.

2. Guazzoni G, Montorsi F, Bocciardi A, Da Pozzo L, Rigatti P, Lanzi R, Pontiroli A. Transperitoneal laparoscopic versus open adrenalectomy for benign hyperfunctioning adrenal tumors: a comparative study. J Urol. 1995; 153(5):1597-1600.

3. Tiberio GA, Baiocchi GL, Arru L, Agabiti Rosei C, De Ponti S, Matheis A, Rizzoni D, et al. Prospective randomized comparison of laparoscopic versus open adrenalectomy for sporadic pheochromocytoma. Surg Endosc. 2008;22(6):1435-1439.

4. Humphrey R, Gray D, Pautler S, Davies W. Laparoscopic compared with open adrenalectomy for resection of pheochromocytoma: a review of 47 cases. Can J Surg. 2008;51(4):276-280.

5. Duh Q, Liu C, Tyrrell JB. Chapter 33. Adrenals. In: Doherty GM, ed. CURRENT Diagnosis \& Treatment: Surgery. 13th ed. New York: McGraw-Hill; 2010.

6. Walther MM, Keiser HR, Choyke PL, Rayford W, Lyne JC, Linehan WM. Management of hereditary pheochromocytoma in von Hippel-Lindau kindreds with partial adrenalectomy. J Urol. 1999;161(2):395-398.

7. Walz MK, Peitgen K, Diesing D, Petersenn S, Janssen OE, Philipp T, Metz KA, et al. Partial versus total adrenalectomy by the posterior retroperitoneoscopic approach: early and long-term results of 325 consecutive procedures in primary adrenal neoplasias. World J Surg. 2004;28(12):1323-1329.

8. Lebuffe G, Dosseh ED, Tek G, Tytgat H, Moreno S, Tavernier B, Vallet B, et al. The effect of calcium channel blockers on outcome following the surgical treatment of phaeochromocytomas and paragangliomas. Anaesthesia. 2005;60(5):439-444.

9. Rubinstein M, Gill IS, Aron M, Kilciler M, Meraney AM, Finelli A, Moinzadeh A, et al. Prospective, randomized comparison of transperitoneal versus retroperitoneal laparoscopic adrenalectomy. J Urol. 2005;174(2):442445; discussion 445 .

10. Vella A, Thompson GB, Grant CS, van Heerden JA, Farley DR, Young WF, Jr. Laparoscopic adrenalectomy for adrenocorticotropin-dependent Cushing's syndrome. J Clin Endocrinol Metab. 2001;86(4):1596-1599.

11. Brauckhoff M, Gimm O, Thanh PN, Bar A, Ukkat J, Brauckhoff K, Bonsch T, et al. Critical size of residual adrenal tissue and recovery from impaired early postoperative adrenocortical function after subtotal bilateral adrenalectomy. Surgery. 2003;134(6):1020-1027; discussion 1027-1028.

12. Parnaby CN, Galbraith N, O'Dwyer PJ. Importance of the adrenal gland blood supply during laparoscopic subtotal adrenalectomy. J Laparoendosc Adv Surg Tech A. 2010;20(4):311-315.

13. Roukounakis N, Dimas S, Kafetzis I, Bethanis S, Gatsulis N, Kostas H, Kyriakou V, et al. Is preservation of the adrenal vein mandatory in laparoscopic adrenal-sparing surgery? JSLS. 2007;11(2):215-218.

14. Liao CH, Chung SD, Lai MK, Yu HJ, Chueh SC. Laparoscopic simultaneous bilateral partial and total adrenalectomy: a longer follow-up. BJU Int. 2009;104(9):12691273 .

15. Nambirajan T, Janetschek G. Laparoscopic partial adrenalectomy. Minim Invasive Ther Allied Technol. 2005;14(2):71-77.

16. Kok KY, Yapp SK. Laparoscopic adrenal-sparing surgery for primary hyperaldosteronism due to aldosteroneproducing adenoma. Surg Endosc. 2002;16(1):108-111.

17. Sasagawa I, Suzuki H, Tateno T, Izumi T, Shoji N, Nakada T. Retroperitoneoscopic partial adrenalectomy using an endoscopic stapling device in patients with adrenal tumor. Urol Int. 1998;61(2):101-103.

18. Disick GI, Munver R. Adrenal-preserving minimally invasive surgery: update on the current status of laparoscopic partial adrenalectomy. Curr Urol Rep. 2008;9(1):67-72.

19. Jeschke K, Janetschek G, Peschel R, Schellander L, Bartsch G, Henning K. Laparoscopic partial adrenalectomy in patients with aldosterone-producing adenomas: indications, technique, and results. Urology. 2003;61(1):69-72; discussion 72.

20. Sasagawa I, Suzuki H, Izumi T, Suzuki Y, Tateno T, Nakada T. Posterior retroperitoneoscopic partial adrenalectomy using ultrasonic scalpel for aldosterone-producing adenoma. J Endourol. 2000;14(7):573-576.

21. Suzuki K, Sugiyama T, Saisu K, Ushiyama T, Fujita K. Retroperitoneoscopic partial adrenalectomy for aldosterone-producing adenoma using an ultrasonically activated scalpel. Br J Urol. 1998;82(1):138-139.

22. Haveran LA, Novitsky YW, Czerniach DR, Kaban GK, Kelly JJ, Litwin DE. Benefits of laparoscopic adrenalectomy: a 10-year single institution experience. Surg Laparosc Endosc Percutan Tech. 2006;16(4):217-221.

23. Shen WT, Lee J, Kebebew E, Clark OH, Duh QY. Selective use of steroid replacement after adrenalectomy: lessons from 331 consecutive cases. Arch Surg. 2006;141(8):771-774; discussion 774-776.

24. Lonser RR, Glenn GM, Walther M, Chew EY, Libutti SK, Linehan WM, Oldfield EH. von Hippel-Lindau disease. Lancet. 2003;361(9374):2059-2067. 